\title{
Associação de mielomeningocele e mielocistocele. Relato de caso
}

Maurus Marques de Almeida Holanda', Christian Diniz Ferreira², Renan Cabral Mota ${ }^{3}$, Rayan Haquim Pinheiro Santos ${ }^{3}$

Hospital Santa Paula, João Pessoa, PB, Hospital Arlinda Marques, João Pessoa, PB e Universidade Federal da Paraíba, João Pessoa, PB, Brasil.

\section{RESUMO}

Relatamos um caso de mielomeningocele dorsolombar associada à mielocistocele na região dorsal, interescapular em recém-nascido do sexo feminino. As correções cirúrgicas dos defeitos foram realizadas nas primeiras 24 horas. São revisadas as teorias de fechamento do tubo neural visando a uma justificativa para a associação dessas malformações.

\section{PALAVRAS-CHAVE}

Anormalidades congênitas, meningomielocele, tubo neural.

\section{ABSTRACT}

Association of myelomeningocele and myelocystocele. Case report

We report a case of dorsal-lumbar myelomeningocele associated with a dorsal myelocistocele in a female newborn. The defects were surgically corrected in the first 24 hours. Revision of the neural tube closure theory was done, to justify the association of these malformation.

\section{KEYWORDS}

Congenital abnormalities, meningomyelocele, neural tube. 


\section{Introdução}

Os defeitos de fechamento de tubo neural (DFTN) são malformações congênitas ou um grupo de anormalidades em que o tubo neural (TN) não se funde em algum ponto do seu comprimento, desde a medula espinhal até o encéfalo. ${ }^{1,2} \mathrm{O}$ desenvolvimento do TN e de suas anomalias ocorre durante a terceira ou quarta semana de gestação, levando ao surgimento de malformações com diversos graus de severidade e prognóstico, associando-se na maioria dos casos a invalidez e morte nos primeiros dias de vida. Estas podem estar associadas também com outras malformações do sistema nervoso central (SNC), como agenesia do corpo caloso, malformação de Chiari, hidrocefalia, hidromielia, diastomielia e seringomielia. $^{2}$

Relatamos um caso de mielomeningocele dorsolombar associada à mielocistocele dorsal, sendo esta uma associação rara, justificando assim a publicação do presente caso.

\section{Relato de caso}

O presente estudo relata o caso clínico de um recém-nascido, do sexo feminino, apresentando uma mielomeningocele dorsolombar associada com uma mielocistocele na região dorsal, interescapular (Figura 1). Não foram encontradas outras malformações associadas.

A genitora não realizou nenhuma consulta pré-natal, inviabilizando assim o diagnóstico intraútero, e não referiu uso de nenhuma medicação sabidamente teratogênica, bem como ausência de suplementação com ácido fólico, durante todo o período gestacional.

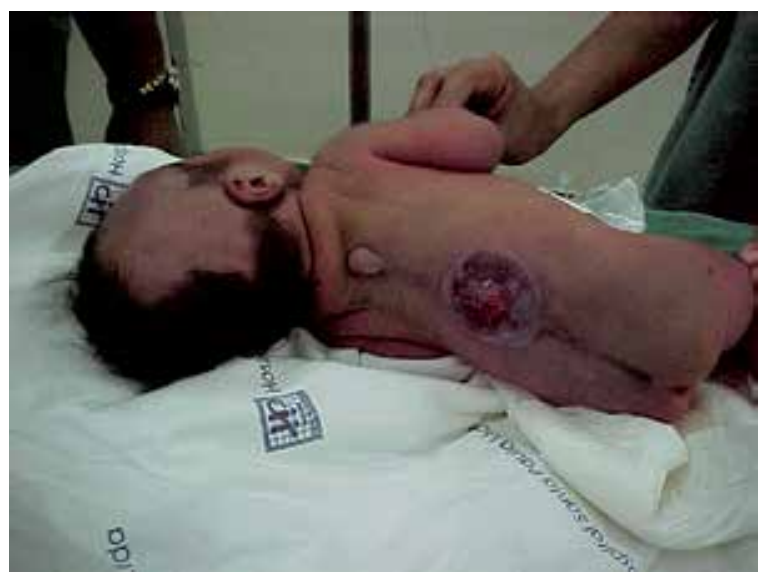

Figura 1 - Mielomeningocele dorsolombar associada à mielocistocele dorsal.
As correções cirúrgicas dos defeitos foram realizadas nas primeiras 24 horas, não havendo intercorrências infecciosas.

Foi corrigida primeiramente a mielomeningocele dorsolombar, de forma usual, com isolamento do placoide nervoso e fechamento hermético da dura-máter coberto por jaquetão de aponeurose lombar (Figura 2). Costumamos expor o saco dural sadio através de pequena laminectomia.

A segunda lesão foi aberta e observada a presença de um segundo cisto, com conteúdo neural, herniado dentro de uma meningocele, caracterizando assim uma mielocistocele, ou um disrafismo cístico espinhal Tipo II, de acordo com a classificação proposta na literatura ${ }^{2}$ (Figura 3). A mielocistocele, por sua vez, foi corrigida, fechada a dura-máter e amputado o conteúdo de pele exuberante.

A criança evoluiu bem, mantendo a flexão dos membros inferiores, antes existente, sem piora do quadro inicial. Acompanhada durante 90 dias, a criança não apresentou hidrocefalia nem deterioração neurológica.

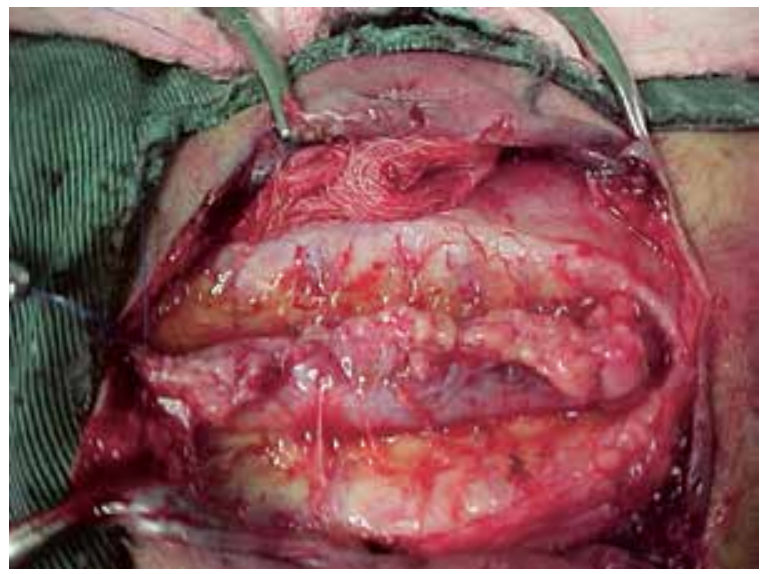

Figura 2 - Correção cirúrgica da mielomeningocele dorsolombar com fechamento da dura-máter.

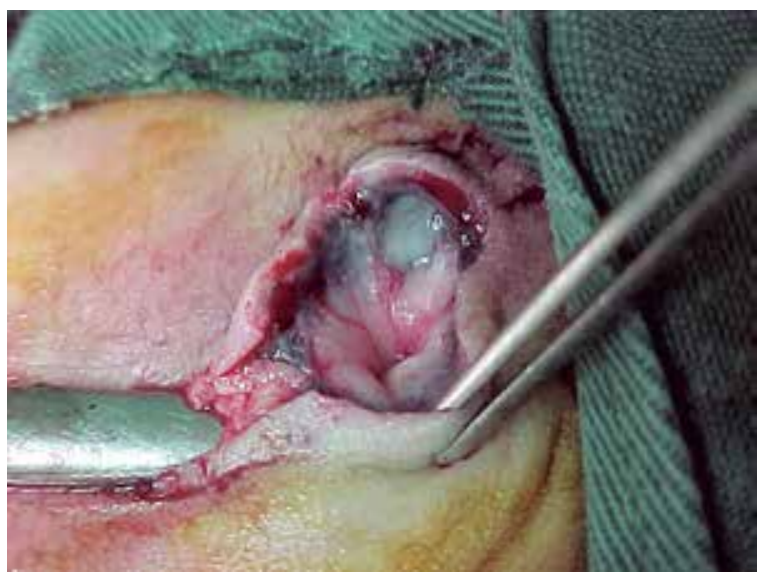

Figura 3 - Mielocistocele exposta durante correção cirúrgica. 


\section{Discussão}

Teorias sobre a patogênese dos defeitos do TN estão relacionadas com o desenvolvimento embrionário do sistema nervoso central (SNC).

No momento, existem duas teorias principais que tentam explicar a gênese do $\mathrm{TN}$ e de suas anomalias associadas. A primeira defende a existência de um fechamento centrífugo e uniformemente progressivo, com a formação dos chamados neuróporos cefálico e caudal, que é a teoria mais utilizada, e a segunda, subsequente, que admite haver um múltiplo fechamento desse tubo através de múltiplos sítios de fusão separados ao longo do dorso do embrião, como ocorre em animais experimentais. . $^{3,4}$

Ainda de acordo com a primeira teoria, o TN inicialmente se fecha na futura região cervical, para, em seguida, fechar-se de forma centrífuga e bidirecionalmente deixando suas extremidades, os neuróporos, abertas. ${ }^{4} \mathrm{O}$ neuróporo superior se fecha no $27^{\circ}$ dia e o inferior se fecha três dias depois. Seria esperado então, baseando-se nessa teoria, que a incidência dos defeitos do TN no ser humano dependesse de qual neuróporo foi afetado, superior ou inferior. ${ }^{3,4}$

Já a segunda teoria preconiza o fechamento do TN através de múltiplos sítios, semelhante ao que acontece em animais experimentais, como ratos e coelhos. ${ }^{5}$ Os sítios de fusão nesses animais são em número de cinco, sendo quatro principais e um secundário. Os sítios são numerados de acordo com a ordem de fechamento: o sítio n-1 começa entre o segundo e o quarto somito, provavelmente no limite entre a medula espinhal e o mielencéfalo, e continua bidirecionalmente. $\mathrm{O}$ sítio $\mathrm{n}$-1, fundindo-se caudalmente, forma o TN torácico (espinha) e o neuróporo caudal. Rostralmente, o sítio $\mathrm{n}-1$ continua até vesículas ópticas (aspecto inferior do rombencéfalo) (Figura 4). O sítio n-2 tem início

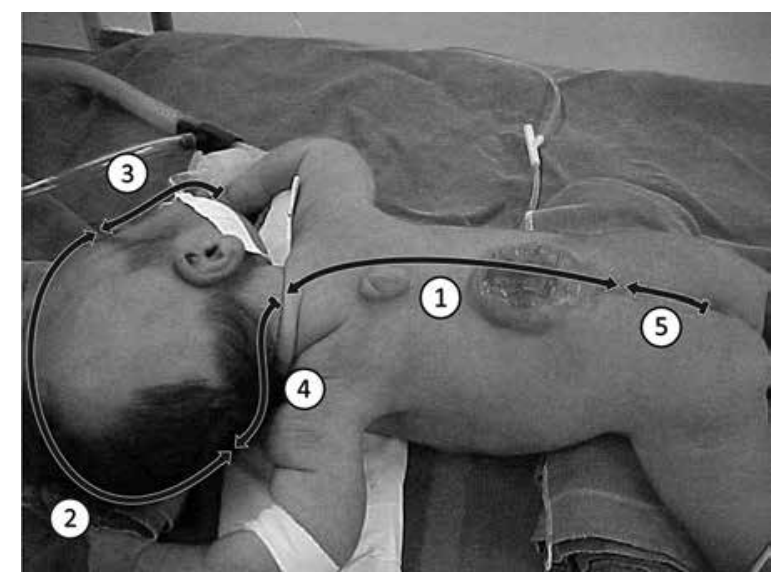

Figura 4 - Esquema representando os sítios de fechamento do tubo neural, segundo teoria de Van Allen. na junção entre o cérebro anterior (prosencéfalo) e o cérebro médio (mesencéfalo). $O$ sítio $n-2$ continua bidirecionalmente, formando dois neuróporos craniais, um na região do prosencéfalo e outro na região do mesencéfalo. Caudalmente, o sítio n-2 progride através do mesencéfalo para terminar no aspecto superior do rombencéfalo. Rostralmente, o sítio n-2 continua através do prosencéfalo até encontrar o sítio n-3 anteriormente.

No mesmo momento da fusão do sítio n-2, o sítio n-3 é iniciado na parte mais rostral da placa neural, adjacente ao estomodeu, prosseguindo-se caudalmente para encontrar o sítio $\mathrm{n}-2$, fechando o neuróporo prosencefálico (neuróporo anterior).

A parte mais rostral das pregas neurais corresponde à posição do lábio superior na face. $\mathrm{O}$ local de junção dos sítios de fechamento n-2 e n-3 corresponde à região interorbitária da face. $O$ sítio n- 4 começa na parte caudal do rombencéfalo de forma unidirecional, caminhando rostralmente para encontrar a parte caudal do sítio n-2, completando o fechamento do TN cranial.

$\mathrm{O}$ quinto sítio se encontra na região sacral logo abaixo do sítio n-1, sendo local de maior frequência de aparecimento de defeitos de fechamento. ${ }^{3}$

Os DFTN mais comuns são a espinha bífida, anencefalia e encefalocele. ${ }^{6}$

As anomalias envolvendo os arcos vertebrais são denominadas de espinha bífida, que pode ser oculta, tratando-se apenas de um achado radiológico, estando algumas vezes associado a um tufo de pelos na região lombossacra, ou cística, associado a meningocele, mielomeningocele e a mielocistocele. . $^{2,6}$

A incidência da espinha bífida cística que agrupa o conjunto de anomalias com protusão de conteúdo é de 1:1000 nascimentos vivos, sendo raro na raça negra. $\mathrm{Na}$ população geral, tem um índice de recorrência de 3\% a $5 \%$ em uma segunda gravidez ou se um dos progenitores é portador desse tipo de malformação. De acordo com a localização, a incidência nas regiões torácicas e toracolombar é de $10 \%$ a $25 \%$, para a região lombar $40 \%$, na região lombossacra $25 \%$ e $10 \%$ para a região sacral, sendo raro o acometimento da região cervical. ${ }^{7}$

A etiologia é multifatorial, com interações entre fatores genéticos e ambientais, como infecções, radiação, diabetes materno, hipertermia e diversas drogas usadas durante a gestação, como o valproato e a carbamazepina. Há uma relação com baixos níveis socioeconômicos, reforçando os fatores nutricionais, como a deficiência de ácido fólico na etiologia dos DFTN. Extensivas investigações têm demonstrado que o ácido fólico antes da concepção e no início da gestação reduziu significativamente a ocorrência e a recorrência de DFTN. ${ }^{8}$

A mielomeningocele ocorre quando as meninges e a medula espinhal herniam através do defeito ósseo produzido pela não fusão dos arcos vertebrais, que pode ou não estar recorberto por uma membrana, íntegra ou 
rota. ${ }^{7,9} \mathrm{~A}$ mielomeningocele é a segunda patologia mais comum do SNC encontrada em crianças e frequentemente está associada com outras anomalias do SNC, além de um comprometimento neurológico acentuado. Aproximadamente $83 \%$ das crianças evoluem com hidrocefalia, e cerca de $74 \%$ já apresentam evidente ventriculomegalia por ocasião do nascimento. Apenas $17 \%$ não desenvolvem dilatação ventricular cirúrgica. ${ }^{9}$

A mielocistocele, também chamada de seringocele ou seringomielocele, refere-se a uma meningocele coberta de pele, com conteúdo de liquor, meninges e um saco herniário internamente que está em continuidade com o canal central da medula, também com liquor e com conteúdo neural. ${ }^{2,4}$ Está associada com um grau variável de hidromielia e muitas vezes com a malformação de Chiari II. ${ }^{10}$ Acredita-se, no entanto, que a mielocistocele e a mielomeningocele façam parte de um espectro resultante de uma mielosquise dorsal limitada. ${ }^{4}$

O diagnóstico pode ser feito no pré-natal por meio de ultrassom, ressonância magnética e da dosagem da alfa-fetoproteína (AFP) no líquido amniótico, entre a $14^{\mathrm{a}}$ e a $16^{\mathrm{a}}$ semana de gestação. ${ }^{4}$

O tratamento cirúrgico da mielomeningocele é realizado precocemente nas 36 horas de vida, visando à correção da malformação e à prevenção de infecções. $\mathrm{O}$ fechamento precoce desse defeito tem aumentado a expectativa de função cognitiva de crianças com mielomeningocele. ${ }^{9}$

Com base em pesquisa realizada na literatura, a associação de mielomeningocele com mielocistocele é rara, sendo encontrados poucos trabalhos em que as duas anomalias estavam associadas. ${ }^{11}$ De acordo com a segunda teoria embriológica citada, essa associação de anomalias aconteceria quando houvesse fechamento defeituoso ou falha na junção dos sítios n-1 e n-5 inferiormente para a mielomeningocele e na junção dos sítios n-1 e n-4 para a mielocistocele.

Tendo em vista as graves anomalias associadas e complicações dos DFTN, faz-se necessária a adoção de medidas de prevenção e intervenção por meio da suplementação de ácido fólico nos alimentos de mulheres em idade fértil antes da concepção e no início da gestação, com objetivo de diminuir a ocorrência e a recorrência dos DFTN.

Outro ponto importante a ser destacado é a presença de poucas teorias que explicam o fechamento do tubo neural. A teoria clássica de fechamento do $\mathrm{TN}$ não explica bem essa associação de DFTN. Por sua vez, a teoria do múltiplo fechamento do tubo neural fornece melhores subsídios para esclarecimento do presente caso, mas, no entanto, não é igualmente satisfatória na explicação dessa associação de anomalias apresentada.

\section{Conflitos de interesse}

Os autores declaram não haver conflitos de interesse.

\section{Referências}

1. Ostos H, Astaiza G, García F, Bautista M, Rojas F, Bermúdez A. Disminución de la incidencia de defectos de cierre del tubo neural en el Hospital Universitario de Neiva; posible efecto de la promoción del consumo de ácido fólico. Revista del Instituto Nacional de Salud. Biomédica (Bogotá). 2000;20(1):18-24.

2. Salomão JF, Cavalheiro $S$, Matushita $H$, Leibinger RD, Bellas AR, Vanazzi E, et al. Cystic spinal dysraphism of the cervical and upper thoracic region. Childs Nerv Syst. 2006;22(3):234-42.

3. Van Allen MI, Kalousek DK, Chernoff GF, Juriloff D, Harris M, McGillivray BC, et al. Evidence for multi-site closure of the neural tube in humans. Am J Med Genet. 1993;47(5):723-43.

4. Klein O, Coulomb MA, Ternier J, Lena G. Cervical myelocystocele: prenatal diagnosis and therapeutical considerations. Childs Nerv Syst. 2009;25(5):523-6.

5. Rotta NT, Aragon de Vecino MC. Malformações congênitas do sistema nervoso central: incidência de cinco anos no Hospital de Clínicas de Porto Alegre. Rev Hosp Clin Porto Alegre. 1989;9(1):10-4.

6. Bizzi JWJ, Silva CE, Vanzin JR, Teixeira LC. Meningomielocele cervical: relato de caso. J Bras Neurocirurg. 2001;12(3);154-7.

7. Martínez de Villarreal LE, Delgado-Enciso I, Valdéz-Leal R, Ortíz-López R, Rojas-Martínez A, Limón-Benavides C, et al. Folate levels and $N(5), N(10)$-methylenetetrahydrofolate reductase genotype (MTHFR) in mothers of offspring with neural tube defects: a case-control study. Arch Med Res. 2001;32(4):277-82.

8. Salomão JF, Leibinger RD, Barbosa APA, Brandão MAP, Cavaleiro $\mathrm{S}$. Mielomeningocele: tratamento cirúrgico, resultados e complicações no primeiro ano de vida. J Bras Neurocirurg. 1997;8(3):93-100.

9. Pang D, Dias MS, Ahab-Barmada M. Split cord malformation: Part I: a unified theory of embryogenesis for double spinal cord malformations. Neurosurgery. 1992;31(3):451-80.

10. Sato K, Yoshida Y, Shirane R, Yoshimoto T. A split cord malformation with paresis of the unilateral lower limb: case report. Surg Neurol. 2002;58(6):406-9.

11. Khandelwal A, Tandon V, Mahapatra AK. An unusual case of 4 level spinal dysraphism: multiple composite type 1 and type 2 split cord malformation, dorsal myelocystocele and hydrocephalous. J Pediatr Neurosci. 2011;6:58-61.

Endereco para correspondência

Maurus Marques de Almeida Holanda

Rua Borja Peregrino, 191 - Centro

58013-342 - João Pessoa, PB, Brasil

E-mail: maurusholanda@hotmail.com 